\title{
A direction finding technique for the ULF electromagnetic source
}

\author{
V. V. Surkov ${ }^{1}$, O. A. Molchanov ${ }^{2}$, and M. Hayakawa ${ }^{3}$ \\ ${ }^{1}$ Moscow Engineering Physics Institute, State University, Moscow, Russia \\ ${ }^{2}$ Institute of Physics of the Earth, Russian Academy of Sciences, Moscow, Russia \\ ${ }^{3}$ The University of Electro-Communications, Chofu Tokyo, Japan
}

Received: 22 June 2004 - Revised: 15 September 2004 - Accepted: 16 September 2004 - Published: 20 September 2004

Part of Special Issue "Precursory phenomena, seismic hazard evaluation and seismo-tectonic electromagnetic effects"

\begin{abstract}
A technique of direction finding is proposed, which can be applied to the magnetic-dipole type source located in the conductive ground. To distinguish a weak ULF source signal from the natural noise a network of multicomponent magnetometers is supposed to be used. The data obtained by the ground-based stations is processed in such a way that a set of partial derivatives of the magnetic perturbations due to the source are found. Comparing these derivatives with theoretical formulae makes it possible, in principle, to find the ULF source parameters such as the distance and amplitude. Averaging the data and a special procedure are proposed in order to exclude random fluctuations in the magnetic moment orientation and to avoid hydrogeological and other local factors.
\end{abstract}

\section{Introduction}

The problem of direction finding of the underground source as well as the problem of searching of a weak electromagnetic signal in the background of natural ionospheric and magnetospheric noise and man-made interference are of a special interest in geophysical studies. For instance, observations of the weak ULF electromagnetic signals before a strong crust earthquakes with magnitude $M>6$ have been reported by a number of authors (e.g. Fraser-Smith et al., 1990; Bernardi et al., 1991; Kopytenko et al., 1990; Molchanov et al., 1992; Hayakawa et al., 1996, 2000; Kawate et al., 1998; Singh et al., 2003; Varotsos et al, 2003a, 2003b). Whether these signals are really associated with tectonic activity and earthquake preparation process have been a subject for recent discussions. In this sense a crucial method in solving the above-mentioned problem could be finding the source signal location.

In order to solve this problem one comes across a number of serious difficulties and complexities. First, the character-

Correspondence to: V. V. Surkov

(surkov@redline.ru) istic wavelength (in vacuum) in the ULF frequency range is so large that an observer is always situated in the near zone, i.e. in such a case the traditional radiowave methods, such as the wave time lag measurement or miscellaneous interference schemes, are inapplicable. Second, in practice, the ULF source of interest is seemingly located under the ground, may be at higher depth. In such a case the electromagnetic signals undergo a strong dissipation and dispersion since the ULF field spreading in conductive layers of the ground is governed by the diffusion law.

In spite of this fact Kopytenko et al. $(2000,2002)$ and Ismaguilov et al. (2003) have proposed a special technique for searching of the underground ULF source. A network of the ground-recording stations equipped with magnetometers was used to detect the time lag or the phase difference between signals recorded at different points. This technique is not reliable enough since the front of signal widens due to the strong dispersion mentioned above. Besides, the typical time-scale of the signals that can be related to earthquake precursor is as large as several tens minutes or hours so that the front of signals is practically absent.

The concept of another technique based on the amplitude difference measurements at different stations is the subject of present study. This technique can be applied just for the cases when the time lag and phase difference are hardly detectable.

\section{Direction finding in the case of two ground-recording stations}

In this section we study a possibility of finding the ULF source in the background of natural noise. Once the distance from the source is much greater than the source size one can use a point-source approximation. In the analysis that follows, we consider a lumped magnetic dipole immersed in a uniform conductor medium. The characteristic spatial scale of the ULF-field generated by the source is supposed to be much greater than the typical size of the ground inhomogeneities. In practice, this scale is of the order of tens or 


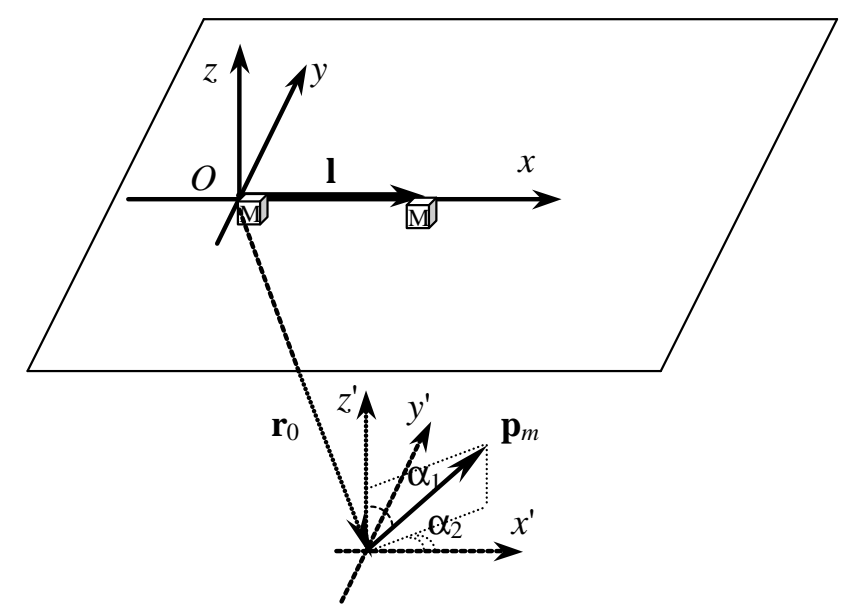

Fig. 1. An illustration of equipment arrangement and the magnetic dipole location.

hundreds kilometers. Hence the mean ground parameters can be applied to study the field distribution.

In the model proposed by Surkov $(1997,1999)$ and Surkov et al. (2003) the effective magnetic moment results from the geomagnetic perturbations due to energization of crack formation in fracture zones in the vicinity of the fault. Acoustic emission of the cracks in conductive layers of the ground excites the geomagnetic perturbations and telluric currents in such a way that the net magnetic moment $\boldsymbol{p}_{m}$ must be pointed oppositely to the vector of geomagnetic induction.

We cannot come close to specifying the origin of the magnetic dipole in any detail, but we consider the case of arbitrary transient magnetic dipole which is immersed in the uniform half-space with constant conductivity $\sigma$. The exact solution of the problem that takes into account the boundary condition at the ground surface have been obtained by Wait and Campbell (1953). It follows from this solution that the ULF electromagnetic field spreading in conductive medium obeys the diffusion law. For example, once the source "turns on" at the moment $t=0$, the electromagnetic perturbations at arbitrary moment $t$ are basically concentrated within area restricted by the radius $r_{d} \sim 2 \sqrt{D t}$, where $D=\left(\mu_{0} \sigma\right)^{-1}$ denotes the coefficient of magnetic diffusion in the conductor medium and $\mu_{0}$ is the magnetic constant. Hence the velocity of the perturbations front propagation can be roughly estimated as $v_{d} \sim \dot{r}_{d} \sim \sqrt{D / t}$.

According to our recent study, the signals possibly associated with impending earthquakes have been detected no farther than $r \sim 100-200 \mathrm{~km}$ from the earthquake epicenter, as documented in many publications (e.g. see a review by Surkov (2000), and references therein, Hayakawa and Molchanov, 2002; Hayakawa and Hattori, 2004). The propagation time for the signals can be estimated as follows $t_{d} \sim r^{2} /(4 D)=\mu_{0} \sigma r^{2} / 4$. Taking the above distances and the conductivity of the upper crust $\sigma=10^{-3} \mathrm{~S} / \mathrm{m}$, we obtain $t_{d} \sim 3-12 \mathrm{~s}$. The characteristic velocity of the perturbation propagation is $v_{d} \sim 2 /\left(\mu_{0} \sigma r\right) \sim 8-16 \mathrm{~km} / \mathrm{s}$. Once the dis- tance between two ground-recording stations is of the order of $l \sim 10 \mathrm{~km}$, the lag time between the onset times is of order $\Delta t \sim \mu_{0} \sigma r l / 2=0.6-1.3 \mathrm{~s}$.

It is usually the case that the duration of the seismogenic signals varies from several tens minutes till hours. The same period of variation, $T$, might be typical for the source itself. Since $T \gg t_{d}$ and $T \gg \Delta t$ the shape of observed signal is practically independent of the propagation time $t_{d}$ and the time lag $\Delta t$. This means that the electromagnetic field of the underground source can be calculated in quasi-stationary approximation. To first order in frequency the exact solution of the problem obtained by Wait and Campbell (1953) is thus transformed to the form

$\boldsymbol{B}=-\frac{\mu_{0}}{4 \pi} \nabla \frac{\boldsymbol{p}_{m} \cdot \boldsymbol{r}}{r^{3}}$,

which coincides with the field of magnetic dipole in the free space, that is the well-known Bio-Savart's law. It is not wonder because in the low-frequency limit the corresponding skin-length tends to infinity. Note that $\boldsymbol{p}_{m}$ in Eq. (1) should be considered as a slowly varying function of time with characteristic period $T \gg t_{d}$.

It is clear that the problem of direction finding cannot be solved in the presence of single ground-recording station and so two stations are necessary at least. The spatial scale of the ULF background noise originated from the ionospheremagnetosphere origin is of the order of hundreds and thousands kilometers. Suppose this spatial scale is much greater than the characteristic length of the perturbations generated by the ULF source. The influence of background noise can then be eliminated by subtraction of the data obtained by two magnetometers located not far from each other. Let the first magnetometer be at the origin $O$ of the coordinate system and the second one is on the $\mathrm{x}$-axis at the distance $l$. The $\mathrm{z}$-axis points upwards.

In geophysical research the source of interest can be situated in the vicinity of a crust fault or in an earthquake hypocenter at higher depth. If the source area is approximately coincides with focal zone, its characteristic size can seemingly be as much as several tens kilometers. In this case the lumped dipole approximation is applied if only the distance from the source is much greater than its characteristic size.

In this study the point magnetic dipole is assumed to be at fixed point with coordinate $\boldsymbol{r}_{0}=\left\{x_{0}, y_{0}, z_{0}\right\}$ to be determined. The direction of the magnetic moment vector $\boldsymbol{p}_{m}$ is defined by two accidental angles $\alpha_{1}$ and $\alpha_{2}$ shown in Fig. 1, so that the magnetic moment projections onto the coordinate axes, $p_{x}, p_{y}$ and $p_{z}$, are random quantities as well.

Assuming for the moment the angles $\alpha_{1}$ and $\alpha_{2}$ are determinate/fixed, the magnetic field of the point moment is given by Eq. (1). If the distance from the moment $r_{0} \gg l$, one can use the approximate formula $\Delta \boldsymbol{B} \approx(\boldsymbol{l} \cdot \nabla) \boldsymbol{B}$ for the magnetometer recording difference. The increment $\Delta \mathbf{B}$ is theoretically expressed through partial derivatives of Eq. (1). Using the abbreviations $\partial_{i}=\partial / \partial_{i}$, we get

$\partial_{i} B_{j}=A\left(a_{i j} p_{x}+b_{i j} p_{y}+c_{i j} p_{z}\right)$, 
where $i=x, y$ and $j=x, y, z$.

Here the following coefficients are used

$a_{x x}=\cos \beta_{1}\left(3-5 \cos ^{2} \beta_{1}\right)$,

$a_{x y}=a_{y x}=b_{x x}=\cos \beta_{2}\left(1-5 \cos ^{2} \beta_{1}\right)$,

$a_{x z}=c_{x x}=\cos \beta_{3}\left(1-5 \cos ^{2} \beta_{1}\right)$,

$a_{y y}=b_{x y}=b_{y x}=\cos \beta_{1}\left(1-5 \cos ^{2} \beta_{2}\right)$,

$a_{y z}=b_{x z}=c_{x y}=c_{y x}=-5 \cos \beta_{1} \cos \beta_{2} \cos \beta_{3}$,

$b_{y y}=\cos \beta_{2}\left(3-5 \cos ^{2} \beta_{2}\right)$,

$b_{y z}=c_{y y}=\cos \beta_{3}\left(1-5 \cos ^{2} \beta_{2}\right)$,

$c_{x z}=\cos \beta_{1}\left(1-5 \cos ^{2} \beta_{3}\right)$,

$c_{y z}=\cos \beta_{2}\left(1-5 \cos ^{2} \beta_{3}\right)$,

$A=\left(\frac{3 \mu_{0}}{4 \pi r_{0}^{4}}\right)^{2}$

The direction cosines,

$\cos \beta_{1}=\frac{x_{0}}{r_{0}}, \cos \beta_{2}=\frac{y_{0}}{r_{0}}, \cos \beta_{3}=\frac{z_{0}}{r_{0}}$,

that give the direction to the source are related as follows,

$\cos ^{2} \beta_{1}+\cos ^{2} \beta_{2}+\cos ^{2} \beta_{3}=1$.

Now we take into account the accidental character of the magnetic moment. In this consideration, the accidental alternating-sign functions in Eq. (2) should be replaced by its mean square values

$$
\begin{aligned}
& \left\langle\left(\partial_{i} B_{j}\right)^{2}\right\rangle=A\left(a_{i j}^{2}\left\langle p_{x}^{2}\right\rangle+b_{i j}^{2}\left\langle p_{y}^{2}\right\rangle+c_{i j}^{2}\left\langle p_{z}^{2}\right\rangle+2 a_{i j} b_{i j}\left\langle p_{x} p_{y}\right\rangle\right. \\
& \left.+2 a_{i j} c_{i j}\left\langle p_{x} p_{z}\right\rangle+2 b_{i j} c_{i j}\left\langle p_{y} p_{z}\right\rangle\right) .
\end{aligned}
$$

Assuming for the moment, there is an equal probability for direction of the magnetic moment vector, and then we get $\left\langle p_{x}^{2}\right\rangle=\left\langle p_{y}^{2}\right\rangle=\left\langle p_{z}^{2}\right\rangle=\left\langle p^{2}\right\rangle /\left\langle p^{2}\right\rangle 3$. The probabilities for the projections are independent with each other in such a way that $\left\langle p_{x} p_{y}\right\rangle=\left\langle p_{x} p_{z}\right\rangle=\left\langle p_{y} p_{z}\right\rangle=0$. Since the source location is assumed to be constant, the angles $\beta_{1}, \beta_{2}$ and $\beta_{3}$ are independent of time. It follows from Eqs. (3)-(5), then

$$
\begin{aligned}
& \left\langle\left(\partial_{x} B_{x}\right)^{2}\right\rangle=A_{1}\left(5 \cos ^{4} \beta_{1}-2 \cos ^{2} \beta_{1}+1\right), \\
& \left\langle\left(\partial_{x} B_{y}\right)^{2}\right\rangle=A_{1}\left(\cos ^{2} \beta_{1}+\cos ^{2} \beta_{2}+5 \cos ^{2} \beta_{1} \cos ^{2} \beta_{2}\right),(6) \\
& \left\langle\left(\partial_{x} B_{z}\right)^{2}\right\rangle=A_{1}\left(\cos ^{2} \beta_{1}+\cos ^{2} \beta_{3}+5 \cos ^{2} \beta_{1} \cos ^{2} \beta_{3}\right),
\end{aligned}
$$

where

$$
A_{1}=\frac{1}{3} A\left\langle p^{2}\right\rangle=3\left\langle p^{2}\right\rangle\left(\frac{\mu_{0}}{4 \pi r_{0}^{4}}\right)^{2} \text {. }
$$

The sets of Eqs. (6) and (4) can be solved for direction cosines

$$
\begin{aligned}
& \cos ^{2} \beta_{1}=\frac{1}{5 a_{1}}\left\{a_{1}+2+\left[\left(a_{1}+2\right)^{2}+5 a_{1}\right]^{1 / 2}\right\}, \\
& \cos ^{2} \beta_{2}=\frac{a_{2}\left(5 \cos ^{4} \beta_{1}-2 \cos ^{2} \beta_{1}+1\right)}{1+5 \cos ^{2} \beta_{1}} .
\end{aligned}
$$

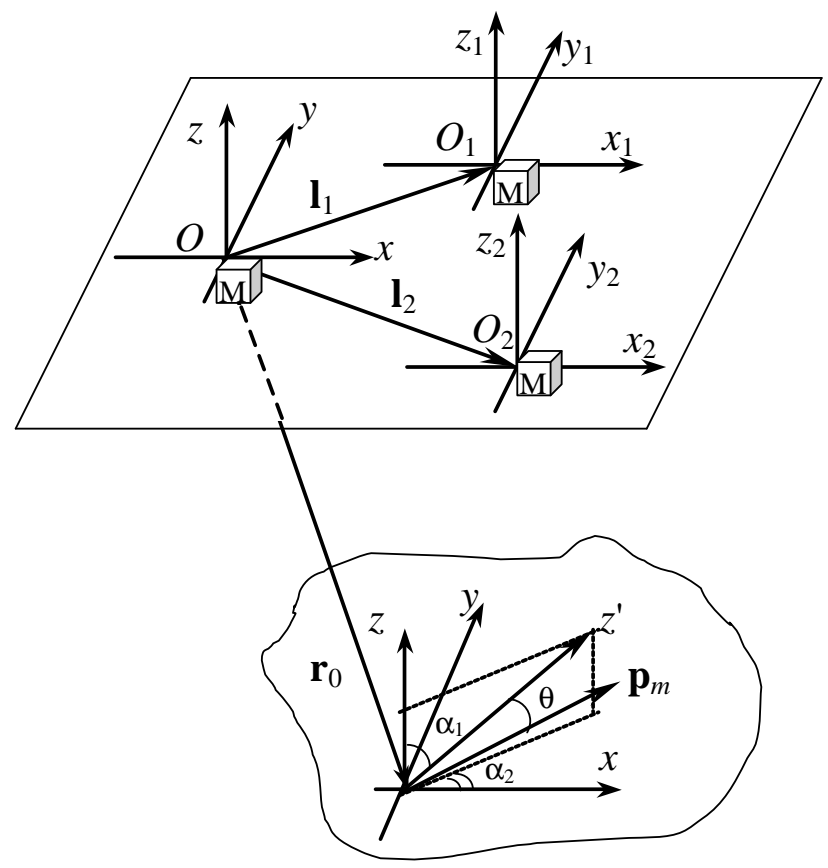

Fig. 2. A schematic drawing of the equipment arrangement and the local coordinate systems. The predominant direction for $\mathbf{p}_{m}$ is shown by $\mathrm{z}^{\prime}$-axis.

Here we made use of the following abbreviations

$a_{1}=1+\frac{\left\langle\left(\partial_{x} B_{y}\right)^{2}\right\rangle+\left\langle\left(\partial_{x} B_{z}\right)^{2}\right\rangle}{\left\langle\left(\partial_{x} B_{x}\right)^{2}\right\rangle}, a_{2}=\frac{\left\langle\left(\partial_{x} B_{y}\right)^{2}\right\rangle}{\left\langle\left(\partial_{x} B_{x}\right)^{2}\right\rangle}$.

The direction cosine $\cos ^{2} \beta_{3}$ is derivable from Eq. (4). The derivatives in Eq. (8) can be evaluated through the average squared differences obtained at different points.

$$
\begin{aligned}
& \left\langle\left(\partial_{x} B_{x}\right)^{2}\right\rangle \approx \frac{1}{l^{2}}\left\langle\left(\Delta B_{x}\right)^{2}\right\rangle,\left\langle\left(\partial_{x} B_{y}\right)^{2}\right\rangle \approx \frac{1}{l^{2}}\left\langle\left(\Delta B_{y}\right)^{2}\right\rangle, \\
& \left\langle\left(\partial_{x} B_{z}\right)^{2}\right\rangle \approx \frac{1}{l^{2}}\left\langle\left(\Delta B_{z}\right)^{2}\right\rangle .
\end{aligned}
$$

Here $l$ is the distance between two three-component magnetometers. Based on Eqs. (7)-(9) and empirical data one can find, in principle, the direction to the source.

Taking into account that the direction cosines must be smaller than unity, we obtain the limitations $a_{1}>5 / 3$ and $a_{2}<1$. It is obvious, if that is not the case, the assumption on homogeneous probability distribution for the direction of $\boldsymbol{p}_{m}$ is not true. In such a case the more complicated technique should be applied.

\section{Direction finding in the case of three ground- recording stations}

Once, in spite of accidental character of the magnetic moment vector $\boldsymbol{p}_{m}$, there is a predominant orientation of the vector $\boldsymbol{p}_{m}$, the use of two magnetometers is insufficient to find the direction cosines. To illustrate this, consider an arrangement in which three three-component magnetometers 
are placed at three different points. One of them is located at the origin of coordinate system $O$, the places of the second and third magnetometers are shown by the vectors $\boldsymbol{l}_{1}$ and $\boldsymbol{l}_{2}$ in Fig. 2. Let z-axis be directed vertically upward while the $\mathrm{x}$-axis points from the West to the East and the y-axis points from the South to the North. For convenience we also use the local Cartesian coordinate systems with their origins at points $O_{1}$ and $O_{2}$. The corresponding axes, $x_{1}, y_{1}, z_{1}$ and $x_{2}, y_{2}, z_{2}$, are chosen to be parallel each other as shown in Fig. 2.

The lumped magnetic dipole $\boldsymbol{p}_{m}$ is characterized by the radius vector $\boldsymbol{r}_{0}=\left\{x_{0}, y_{0}, z_{0}\right\}$ and its orientation is random (Fig. 2). Making allowance for the inequality $\left|\boldsymbol{l}_{k}\right| \ll\left|\boldsymbol{r}_{0}\right|$ where $k=1,2$, one can use the approximate relationship $\Delta \boldsymbol{B}_{k} \approx\left(\boldsymbol{l}_{k} \cdot \nabla\right) \boldsymbol{B}_{k}$. Hence we get

$$
\Delta B_{i}^{(k)}=l_{x}^{(k)} \partial_{x} B_{i}^{(k)}+l_{y}^{(k)} \partial_{y} B_{i}^{(k)},
$$

where $l_{x}^{(k)}$ and $l_{y}^{(k)}$ are the projections of the vectors $\boldsymbol{l}_{k}$ onto the coordinate axes. The set of linear equations (10) can be solved for the partial derivatives and their mean squared values are

$$
\begin{gathered}
\left\langle\left(\partial_{x} B_{i}\right)^{2}\right\rangle=\frac{1}{\Delta^{2}}\left\langle\left(\Delta B_{i}^{(1)} l_{y}^{(2)}-\Delta B_{i}^{(2)} l_{y}^{(1)}\right)^{2}\right\rangle \\
\left\langle\left(\partial_{y} B_{i}\right)^{2}\right\rangle=\frac{1}{\Delta^{2}}\left\langle\left(\Delta B_{i}^{(2)} l_{x}^{(1)}-\Delta B_{i}^{(1)} l_{x}^{(2)}\right)^{2}\right\rangle \\
\Delta=l_{x}^{(1)} l_{y}^{(2)}-l_{x}^{(2)} l_{y}^{(1)}, i=x, y, z .
\end{gathered}
$$

It should be noted that in the atmosphere only five partial derivatives of $\boldsymbol{B}$ among the nine are independent values since there are four connections between the derivatives following from the Maxwell's equations $\nabla \times \boldsymbol{B}=0$ and $\nabla \cdot \boldsymbol{B}=0$. In our case there is the connection $\partial_{y} B_{x}=\partial_{x} B_{y}$. It follows from this,

$$
\Delta B_{x}^{(1)} l_{x}^{(2)}-\Delta B_{x}^{(2)} l_{x}^{(1)}=\Delta B_{y}^{(2)} l_{y}^{(1)}-\Delta B_{y}^{(1)} l_{y}^{(2)},
$$

When substituting the experimental data in Eq. (12), one may expect that this equation will hold only approximately. Nevertheless, Eq. (12) may be used for the control of recording accuracy.

The main idea of the method proposed in the present paper is that the use of the experimental differences $\Delta B_{i}^{(k)}$ makes it possible to evaluate the mean square of the tensor of partial derivatives $\left\langle\left(\partial_{i} B_{j}\right)^{2}\right\rangle$ in Eq. (11). Equating these experimental derivatives to that given by Eqs. (3) and (5), we can estimate the mean magnetic dipole projections. On the other hand, these mean projections can be calculated theoretically.

In order to estimate the averaged magnetic dipole projections in Eq. (5) we suppose that there is a predominant direction for the vector $\boldsymbol{p}_{m}$ despite of the accidental orientation of the magnetic dipole. This direction shown in Fig. 2 by z'axis is defined by the constant angles $\alpha_{1}$ and $\alpha_{2}$. The probability density distribution around $\mathrm{z}^{\prime}$-axis is assumed to be axially symmetric so that the direction of $\boldsymbol{p}_{m}$ depends solely on the polar angle $\theta$ between the vector $\boldsymbol{p}_{m}$ and $\mathrm{z}^{\prime}$-axis. This probability distribution can be characterized by the two mean squared projections of $\boldsymbol{p}_{m}$, parallel and perpendicular to $\mathrm{z}^{\prime}$ axis; that is

$\left\langle p_{\|}^{2}\right\rangle=\left\langle p^{2} \cos ^{2} \theta\right\rangle$ and $\left\langle p_{\perp}^{2}\right\rangle=\left\langle p^{2} \sin ^{2} \theta\right\rangle$.

The mean projections of the magnetic moment in Eq. (5) can be expressed through the mean values (Eq. 13) as follows

$$
\begin{aligned}
& \left\langle p_{x}^{2}\right\rangle=0.5\left\langle p_{\perp}^{2}\right\rangle+p_{\text {as }} \sin ^{2} \alpha_{1} \cos ^{2} \alpha_{2}, \\
& \left\langle p_{y}^{2}\right\rangle=0.5\left\langle p_{\perp}^{2}\right\rangle+p_{\text {as }} \sin ^{2} \alpha_{1} \sin ^{2} \alpha_{2}, \\
& \left\langle p_{z}^{2}\right\rangle=0.5\left\langle p_{\perp}^{2}\right\rangle+p_{\text {as }} \cos ^{2} \alpha_{1}, \\
& \left\langle p_{x} p_{y}\right\rangle=p_{\text {as }} \sin ^{2} \alpha_{1} \sin \alpha_{2} \cos \alpha_{2}, \\
& \left\langle p_{x} p_{z}\right\rangle=p_{\text {as }} \sin \alpha_{1} \cos \alpha_{1} \cos \alpha_{2}, \\
& \left\langle p_{y} p_{z}\right\rangle=p_{\text {as }} \sin \alpha_{1} \cos \alpha_{1} \sin \alpha_{2},
\end{aligned}
$$

where the parameter

$p_{\text {as }}=\left\langle p_{\|}^{2}\right\rangle-0.5\left\langle p_{\perp}^{2}\right\rangle$

takes into account the asymmetry of the probability density distribution. In particular, if all the directions of the vector $\boldsymbol{p}_{m}$ have equal probability, then $p_{\text {as }}=0$.

Substituting a set of Eq. (14) into Eq. (5), and using a set of Eq. (11), we come to a set of six equations for eight unknown parameters $\alpha_{1}, \alpha_{2}, \beta_{1}, \beta_{2}, \beta_{3}, p_{\text {as }},\left\langle p_{\perp}^{2}\right\rangle$ and $A$. As we have noted above, only five equations among them are independent. These equations should be supplemented by the connection (Eq. 4). Nevertheless we need some additional information for solving the problem.

It is worth mentioning that in some theoretical models the crack's acoustic emission due to rock fracture gives rise to formation of the electric currents and geomagnetic perturbations, whose effective magnetic moment points oppositely to the vector of local geomagnetic field $\boldsymbol{B}_{0}$ (Surkov, 1997, 1999; Surkov et al., 2003). In such a case the angles $\alpha_{1}$ and $\alpha_{2}$ can be considered as given values since the total magnetic moment of whole crack ensemble is directed oppositely to the vector $\boldsymbol{B}_{0}$. Note that the problem can also be solved when the asymmetry parameter is small enough. The same technique can be developed for the ULF electric current moment/dipole.

One of the challenges of the direction finding problem is to know enough the influence of the systematic errors on the results of measurements. For example, the magnetometer antennas cannot be quite co-directed. Besides it is possible to think about the distortions of local Earth's electromagnetic field caused by the relief features, meteorological and hydrogeological factors, local variations of the ground conductivity and etc.

In order to eliminate the influence of these and other factors the data obtained in points $O_{1}$ and $O_{2}$ should be preliminary corrected with respect to a reference magnetometer placed in the point $O$. For example it is customary to correct these data, $\boldsymbol{B}_{1}$ and $\boldsymbol{B}_{2}$, as follows

$$
\boldsymbol{B}^{\prime}{ }_{1}=\hat{\mathbf{A}}_{1} \times \boldsymbol{B}_{1}, \quad \boldsymbol{B}^{\prime}{ }_{2}=\hat{\mathbf{A}}_{2} \times \boldsymbol{B}_{2},
$$

where $\hat{\mathbf{A}}_{1}$ and $\hat{\mathbf{A}}_{2}$ are the symmetric matrixes, whose coefficients are selected empirically in such a way that the quantities $\boldsymbol{B}^{\prime}{ }_{1}$ and $\boldsymbol{B}^{\prime}{ }_{2}$ agree with each other and with the reference 
magnetometer recording on average. In fact these matrixes slightly turn the vectors of magnetic induction in order to make the recordings compatible with each other. When calculating the differences $\Delta B_{i}^{(k)}$ in Eq. (11), these corrected values $\boldsymbol{B}^{\prime}{ }_{1}$ and $\boldsymbol{B}^{\prime}{ }_{2}$ should be used.

\section{Conclusions}

This promising technique based on evaluation of the partial derivatives of the magnetic perturbations makes it possible, in principle, to find both the location of the ULF magneto-dipole source and the direction cosines of the mean moment vector. The technique can be applied irrespective of the fact that either the remote source is situated in the ground, in the atmosphere or the ionosphere. In addition, this technique is valid when the signals from underground ULF source undergo strong dissipation and dispersion in conductive rock that makes difficulties in recording of time lag and phase difference of the signals. From this viewpoint, the analyses of amplitudes and spatial derivatives of the magnetic perturbations, seems, to be more perspective. There are two limitations of the technique, first, a spatial scale of natural noise variation should be much greater than both characteristic length of the ULF signal variation and distances between magnetometers, and second, the characteristic source size must be small compared to distance from the source. As both conditions are satisfied, the technique allows us to discriminate a weak useful ULF signal from background noise with confidence. This information can be extremely useful for interpretation of experimental data and for understanding of the origin the ULF signals and, in particular, for finding the electromagnetic signals possibly related to impending earthquakes.

Edited by: P. F. Biagi

Reviewed by: two referees

\section{References}

Bernardi, A., Fraser-Smith, A. C., McGill, P. R., and Villard, Jr. O. G.: ULF magnetic field measurements near the epicenter of the $M_{S}$ 7.1 Loma Prieta earthquake, Phys. Earth Planet. Inter., 68, 45-63, 1991.

Fraser-Smith, A. C., Bernardi, A., McGill, P. R., Ladd, M. E., Helliwell, R. A., and Villard, O. G. Jr.: Low-frequency magnetic field measurements near the epicenter of the $M_{S} 7.1$ Loma Prieta earthquake, Geophys. Res. Lett., 17, 1465-1468, 1990.

Hayakawa, M., Kawate, R., Molchanov, O. A., and Yumoto, K.: Results of ULF magnetic field measurements during the Guam earthquake of 8 August 1993, Geophys. Res. Lett., 23, 241-244, 1996.

Hayakawa, M., Itoh, T., Hattori, K., and Yumoto, K.: ULF electromagnetic precursors for an earthquake at Biak, Indonesia on 17 February 1996, Geophys. Res. Lett., 27, 1531-1534, 2000.

Hayakawa, M. and Molchanov, O. A., editors: "Seismo Electromagnetics: Lithosphere-Atmosphere-Ionosphere Coupling", TERRAPUB, 477 p., Tokyo, 2002.
Hayakawa, M. and Hattori, K.: ULF electromagnetics emissions associated with earthquakes: Review, Trans. Fundamentals and Materials, Special Issue on Electromagnetic Theory and Its Application Dec., in press, 2004.

Ismaguilov, V. S., Kopytenko, Yu. A., Hattori, K., and Hayakawa, M.: Variations of phase velocity and gradient of ULF geomagnetic disturbances connected with the Izu strong earthquakes, Nat. Haz. Earth Sys. Sci., 3, 211-215, 2003, SRef-ID: 1684-9981/nhess/2003-3-211.

Kawate, R., Molchanov, O. A., and Hayakawa, M.: Ultra-lowfrequency magnetic fields during the Guam earthquake of $8 \mathrm{Au}-$ gust 1993 and their interpretation, Phys. Earth Planet. Inter., 105, 229-238, 1998.

Kopytenko, Yu. A., Matiashvili, T. G., Voronov, P. M., Kopytenko, E. A., and Molchanov, O. A.: Detection of ULF emissions connected with the Spitak earthquake and its aftershock activity, based on geomagnetic pulsation data at Dusheti and Vardzia observations, Preprint of IZMIRAN, 1990 (in Russian).

Kopytenko, Yu. A., Ismaguilov, V. S., Kopytenko, E. A., Voronov, P. M., and Zaitsev, D. B.: Magnetic location of geomagnetic disturbance sources, DAN, series Geophysics, 371, 685-687, 2000.

Kopytenko, Yu. A., Ismaguilov, V. S., Molchanov, O. A., Kopytenko, E. A., Voronov, P. M., Hattori, K., Hayakawa, M., and Zaitsev, D. B.: Investigation of ULF magnetic disturbances in Japan during seismic active period, J. Atm. Electr., 22, 207-215, 2002.

Molchanov, O. A., Kopytenko, Yu. A., Voronov, P. M., Kopytenko, E. A., Matiashvili, T. G., Fraser-Smith, A. C., and Bernardi, A.: Results of ULF magnetic field measurements near the epicenters of the Spitak $\left(M_{s}=6.9\right)$ and Loma Prieta $\left(M_{s}=7.1\right)$ earthquakes: comparative analysis, Geophys. Res. Lett., 19, 14951498, 1992.

Singh, R. P., Singh, B., Mishra, P. K., and Hayakawa, M.: On the lithosphere-atmosphere coupling of seismo-electromagnetic signals, Radio Sci., 38, 1065-1074, 2003.

Surkov, V. V.: The nature of electromagnetic forerunners of earthquakes, Transactions (Doklady) of the Russian Academy of Sciences. Earth Science Sections, 355, 945-947, 1997.

Surkov, V. V.: ULF electromagnetic perturbations resulting from the fracture and dilatancy in the earthquake preparation zone, in "Atmospheric and Ionospheric Phenomena Associated with Earthquakes", edited by Hayakawa, M., TERRAPUB, Tokyo, 357370, 1999.

Surkov, V. V.: Electromagnetic effects caused by earthquakes and explosions. MEPhI press, Moscow, 2000, 448 pp. (in Russian).

Surkov, V. V., Molchanov, O. A., and Hayakawa, M.: Preearthquake ULF electromagnetic perturbations as a result of inductive seismomagnetic phenomena during microfracturing, J. Atmos. Solar-Terr. Phys., 65, 31-46, 2003.

Varotsos, P. A., Sarlis N. V., and Skordas, E. S.: Long-range correlations in the electric signals that precede rupture: Further investigations, Phys. Rev., E67, 021109, 2003.

Varotsos, P. A., Sarlis N. V., and Skordas, E. S.: Attempt to distinguish electric signals of a dichotomous nature, Phys. Rev., E68, 031106, 2003.

Wait, J. R. and Campbell, L. L.: The field of an oscillating magnetic dipole immersed in a semi-infinite conducting medium, J. Geophys. Res., 58, 167-178, 1953. 